\title{
Dust in Evolving Galaxies
}

\author{
Paola Andreani \\ Oss. Astr. di Padova, v.lo dell'Osservatorio 5, I-35122 Padova, Italy \\ Max-Planck I. f. Extraterrestrische Physik, Garching, Germany
}

\begin{abstract}
I will first review the observational evidence relating dust emission and the energy production in the far-IR/submm range. This latter contains crucial information on the global baryons transformation and the related stellar activity in the Universe. Present knowledge on this topic relies mainly on the far-IR local surveys of IRAS and ISO missions and on submm/mm surveys performed with SCUBA and MAMBO arrays. Further constraints are provided by the measurements of the Cosmic far-IR Background (CFIRB). Our scanty knowledge of galaxy formation and evolution is mainly caused by the difficulties of unveiling stellar activity at redshifts larger than 1 and at present we may only have detected massive objects in a transient hyper-luminous phase. We still lack an unbiased census of the much more numerous population of lower luminosity dusty objects. It will soon be possible to disclose the entire history of evolving dusty objects, and therefore of the stellar activity, selecting unbiased samples out of far-IR imaging and photometry in deep far-IR surveys.
\end{abstract}

\section{Dust Emission as Star-Formation Tracer}

In the last twenty years, observational evidence has been accumulating that the most important episodes of star-formation in the local Universe and up to $z=1$ happen in dusty and gaseous environments. But also at $z>1$ there are indirect indications of an important role of dust: the existence of hyper-luminous high $z$ IR galaxies, the large amounts of processed material (dust and metals) in quasars and radio galaxies, the occurrence of dust extinction in optically selected galaxies at $z=2 \div 4$ (hence biased against dust obscuration), the presence of an integrated emission, in the form of a far-IR/submm isotropic background corresponding to a very energetic high- $z$ phase, and the population of energetic submm $/ \mathrm{mm}$ sources with very large star-formation rate $\left(\sim 10^{2} M_{\odot} \mathrm{yr}^{-1}\right)$.

\subsection{Constraints on the stellar activity history from the IR energy production}

It is possible to trace back the stellar activity history by studying the evolution of the IR radiation production (Gispert, Lagache \& Puget 2000). Current observations provide useful constraints on this history: 
- At low redshifts $z<1$ local IR surveys (IRAS and ISO) showed a population of high luminosity objects, with no or very faint optical counterpart, corresponding to merging or interacting systems for which the ratio between the IR to the optical luminosity, $\frac{\mathrm{L}_{\mathrm{FIR}}}{\mathrm{L}_{\mathrm{opt}}}$, increases as the bolometric luminosity increases. ISO deep surveys showed how the IR energy production strongly increases in the redshift range between 0 and 1, with deep galaxy counts significantly exceeding the non-evolving predictions.

- At redshifts between $z=2$ and $z=4$ measurements of the CFIRB constrain well the energy production and the number of sources giving origin to the CFIRB while present surveys are not sensitive enough.

(a) The large value of the ratio, $\frac{E(F I R)}{E(\text { opt })}=1-2.6$, between the background energy content in the far-IR (at $\lambda \geq 6 \mu m$ ) and that in the optical $(\lambda<6 \mu \mathrm{m})$ with respect to that measured in the local systems, $\frac{E(F I R)}{E(\text { opt })}=0.3$, means that the galaxy population responsible for the stellar activity underwent a major change in its properties.

(b) The energy content at $15 \mu \mathrm{m}$ is nearly as large as in the optical. But the background at $15 \mu \mathrm{m}$ is made up of a very small number of objects compared to the number of sources responsible for the optical background.

(c) The spectrum of the CFIRB at long wavelengths is significantly flatter than that of individual IR galaxies. Therefore the submm part of the CFIRB cannot be due to the same population that gives rise to the background at $150 \mu \mathrm{m}$ and contains unique information about the high-redshift population.

- Current submm $/ \mathrm{mm}$ surveys address issues to the stellar activity at high- $z$ because of the shift of the thermal emission spectrum into their wavelength bands. SCUBA surveys detected high-luminosity objects with a large starformation rate (>200 $\left.M_{\odot} \mathrm{yr}^{-1}\right)$. The faint submm counts significantly exceed a no-evolution model requiring roughly $(1+z)^{3}$ luminosity evolution out to $z \sim 2$, but with poor constraints at higher redshifts.

In summary, star-formation activity, as detected in the far-IR, appears in total at least comparable to all that seen unobscured in the UV.

\section{Lower Luminosity Dusty Objects}

The outlined observational status suggests that the bulk of the CFIRB is very likely due to a small number of energetic sources (ISOCAM galaxies at $15 \mu \mathrm{m}$, SCUBA galaxies at $850 \mu \mathrm{m}, \ldots$ ) with a possible $20 \%$ contribution of AGN activity caused by dust enshrouded AGN + star-formation, which could have been higher in the past. These objects alone, however, fail to reproduce the optical/UV background, and are linked to massive dust distributions and/or large systems in advanced stages of chemical evolution and in an accelerated phase of evolution. Their evolutionary pattern is quite different from that expected for disc-dominated galaxies, which form stars quiescently and continuously during a large fraction of Hubble time. 
Where and what is this much more numerous population of lower luminosity dusty objects? As discussed below only future far-IR surveys will address this issue in a complete and unbiased way.

\subsection{Do Extremely Red Objects hide a dusty population?}

High- $z$ dusty galaxies can be singled out among candidates selected according to their extreme red colours $(5<R-K<8)$. The nature of these extreme red objects (EROs) is still controversial (see Cimatti et al. 2000 and references therein), their large red colours being due to either a passively evolving population of stars in old $L_{*}$ ellipticals at $z>1$ or to strongly extincted starbursts or AGNs whose UV-optical light is reddened by dust.

In principle it is possible to distinguish the two classes performing (1) nearIR spectroscopy to detect either emission lines $(\mathrm{H} \alpha)$ of ongoing star-formation or sharp spectral breaks due to old stellar populations; (2) submm/mm observations to detect dust thermal emission and radio observations to detect that part of the synchrotron emission correlated with star-formation; (3) high spatial resolution optical and near-IR images to study the morphology, which is expected to be regular, compact and central peaked for old ellipticals and irregular, asymmetric and distorted for dusty starbursts.

Because of the intrinsic difference of their spectral energy distribution, candidate dusty objects can be selected, in the redshift range $1<z<2$, from their IR colours on the plane (I-K),(J-K) (Pozzetti \& Mannucci 2000).

Follow-up observations of the thermal emission is mandatory to confirm their nature (Cimatti et al., in progress). Here we report some results on the dusty class.

- HR10 is so far the only targeted ERO with a measured spectroscopic redshift (Graham \& Dey 1996). Submm/mm photometry and spectroscopy undoubtedly proved that this object is highly obscured, has a large thermal far-infrared luminosity, high star-formation rate $\left(\sim 700 M_{\odot} \mathrm{yr}^{-1}\right)$ and a large molecular gas mass $M\left(\mathrm{H}_{2}\right)\left(1.6 \times 10^{11} h_{50}^{-2} M_{\odot}\right)$ (Cimatti et al. 1998; Dey et al. 1999; Andreani et al. 2000).

- EROs have been found among submm-selected objects (Smail et al. 1999; Gear et al. 2000) with plausible redshifts between 2 and 4, implying that they could represent $10 \%$ of the faint submm population.

- Submm/mm search for dusty emission in other 30 EROs suggests that $30 \%$ of the entire ERO population is dusty, with the reddest objects being more likely the more dusty ones.

\section{Future research}

Since it is becoming more and more evident that the assembly of galaxies is taking place between redshift $z=1$ and $z=3$, where a peak of activity is detected, to unveil the nature of the expected numerous dusty population at this epoch the goal of future surveys must be: 
- measure the redshifted dust continuum and/or non-thermal emission from forming objects in as many colours as possible,

- produce high-spatial resolution maps to reduce the large uncertainty on source identification because of (1) large positional error bars and (2) lack of optical/near-IR counterpart,

- estimate the nature of the ionizing continua (e.g. nonthermal AGN versus thermal starburst continuum) with low- and high-excitation atomic and ionic fine-structure lines.

- measure redshifts, star formation efficiency (star formation per unit gas mass) in high redshift galaxies, dynamics and mass of the gas via the far$\mathrm{IR} /$ submm [CI], [CII], [NII], [OI], [OIII] fine structure lines and molecules, CO.

Surveys must be carefully planned and all the statistical biases and observational limitations must be studied. The reliability of the results must be checked through Monte Carlo simulations and a large number of mock surveys must be built to determine what happens when a selection of a sample is made. This provides information on the observational biases introduced by the observing procedures and allow to understand how the different parameters affect the source observation and the detection.

This approach was followed by us to plan surveys with the PACS and SPIRE Instruments aboard of the FIRST Satellite. The approach we used is as empirical as possible and uses a statistical analysis. It is generally called Backward Evolution since it starts from the present epoch and tries to extrapolate the local (statistical) properties of galaxies to higher redshifts to predict how sources formed, behaved and evolved since their birth. The statistical properties of the evolving underlying population of objects are inferred from the luminosity function and its evolution, the spatial distribution of source via the angular correlation function, the integral and differential source counts and the integrated background due to the integrated emission from the unresolved sources.

Acknowledgments. The author acknowledges support from the Alexander von Humboldt and warmly thanks MPE for hospitality.

\section{References}

Andreani, P., Cimatti, A., Loinard, L., Röttgering, H. 2000, A\&A, 354, L1

Cimatti, A., Andreani, P., Röttgering, H., Tilanus, R. 1998, Nat, 392, 895

Cimatti, A., et al. 2000, Proc. SPIE Vol. 4005, 45, Ed. J. Bergeron

Dey, A. et al. 1999, ApJ, 519, 610

Gear, W.K., Lilly, S.J., Ivison, R.J., Smail, I., Wright, G.S., Liu, M.C. 2000, MNRAS, 316, L51

Gispert, R., Lagache, G., Puget, J.L. 2000, A\&A, 360, 1

Graham, J.R., Dey, A. 1996, ApJ, 471, 720

Pozzetti, L., Mannucci, F. 2000, MNRAS, in press

Smail, I., et al. 1999, MNRAS, 308, 1061 